\title{
Erratum to: Effect of simvastatin plus cetuximab/irinotecan for $K R A S$ mutant colorectal cancer and predictive value of the $R A S$ signature for treatment response to cetuximab
}

\author{
Jeeyun Lee • Yong Sang Hong • Jung Yong Hong • Se Won Han • Tae Won Kim • \\ Hye Jin Kang • Tae You Kim • Kyu-pyo Kim • Suk Hyung Kim • In-Gu Do • \\ Kyoung-Mee Kim • Insuk Sohn • Se Hoon Park • Joon Oh Park • Ho Yeong Lim • \\ Yong Beom Cho • Woo Yong Lee • Seong Hyeon Yun • Hee Cheol Kim • Young Suk Park • \\ Won Ki Kang
}

Published online: 20 September 2014

(C) Springer Science+Business Media New York 2014

Erratum to: Invest New Drugs (2014) 32:535-541

DOI 10.1007/s10637-014-0065-x

In the original version of this article, we have had unintentional mistakes for measuring progression-free survival (PFS). The corrected progression-free survival was 3.7 months $(95 \%$ CI 2.1-5.3). So, we revised Table 3, Figure 2a, c and manuscript (Results section and Discussion section). We also added secondary endpoints which had been described in the study (Merck). protocol according to the recommendation by the sponsor

Table 3 Response according to RECIST (version 1.1) and survival outcome

\begin{tabular}{ll}
\hline Response & $\begin{array}{c}\text { Number of patients } \\
(\mathbf{\%}, \mathbf{9 5} \% \mathbf{C I})\end{array}$ \\
Complete response & $0(0)$ \\
Confirmed partial response & $1(1.9,-1.8-5.6)$ \\
Confirmed stable disease & $33(63.5,50.4-76.6)$ \\
Progressive disease & $13(25.0,13.2-36.8)$ \\
Not evaluable & $5(9.6,1.6-17.6)$ \\
Disease control rate $(\mathrm{CR}+\mathrm{PR}+\mathrm{SD})$ & $\mathbf{3 4}(\mathbf{6 5 . 4 , 5 2 . 5} \mathbf{- 7 8 . 3})$ \\
Survival outcome & Months $\mathbf{( 9 5 \%} \mathbf{C I})$ \\
Progression-free survival & $\mathbf{3 . 7}(\mathbf{2 . 1 - 5 . 3 )}$ \\
3-month progression-free survival rate & $\mathbf{5 5 \%}$ \\
6-month progression-free survival rate & $\mathbf{3 3 \%}$ \\
1-year progression-free survival rate & $\mathbf{0 \%}$ \\
Overall survival - months & $12.8(9.5-16.2)$ \\
6-month overall survival rate & $81 \%$ \\
1-year overall survival rate & $65 \%$
\end{tabular}

The online version of the original article can be found at http://dx.doi.org/ 10.1007/s10637-014-0065-x.

J. Lee · J. Y. Hong · S. H. Park · J. O. Park · H. Y. Lim • Y. S. Park · W. K. Kang $(\bowtie)$

Division of Hematology-Oncology, Department of Medicine, Samsung Medical Center, Sungkyunkwan University School of Medicine, 50 Irwon-dong Gangnam-gu, Seoul 135-710, South Korea

e-mail: wkkang@skku.edu

Y. S. Hong • T. W. Kim $・$ K.-p. Kim

Department of Oncology, Asan Medical Centre, University of Ulsan College of Medicine, Seoul, South Korea

S. W. Han • T. Y. Kim

Division of Medical Oncology, Department of Internal

Medicine, Seoul National University Hospital, Seoul National

University College of Medicine, Seoul, South Korea

\section{H. J. Kang}

Division of Hematology/Oncology, Department of Internal Medicine, Korea Cancer Center Hospital, Korea Institute of Radiological and Medical Sciences, Seoul, South Korea

\section{S. H. Kim・I.-G. Do • K.-M. Kim}

Department of Pathology, Samsung Medical Center, Sungkyunkwan

University School of Medicine, Seoul, South Korea

I. Sohn

Samsung Medical Center, Samsung Cancer Research Institute,

Seoul, South Korea

Y. B. Cho $\cdot$ W. Y. Lee $\cdot$ S. H. Yun $\cdot$ H. C. Kim

Department of Surgery, Samsung Medical Center, Sungkyunkwan

University School of Medicine, Seoul, South Korea 
a

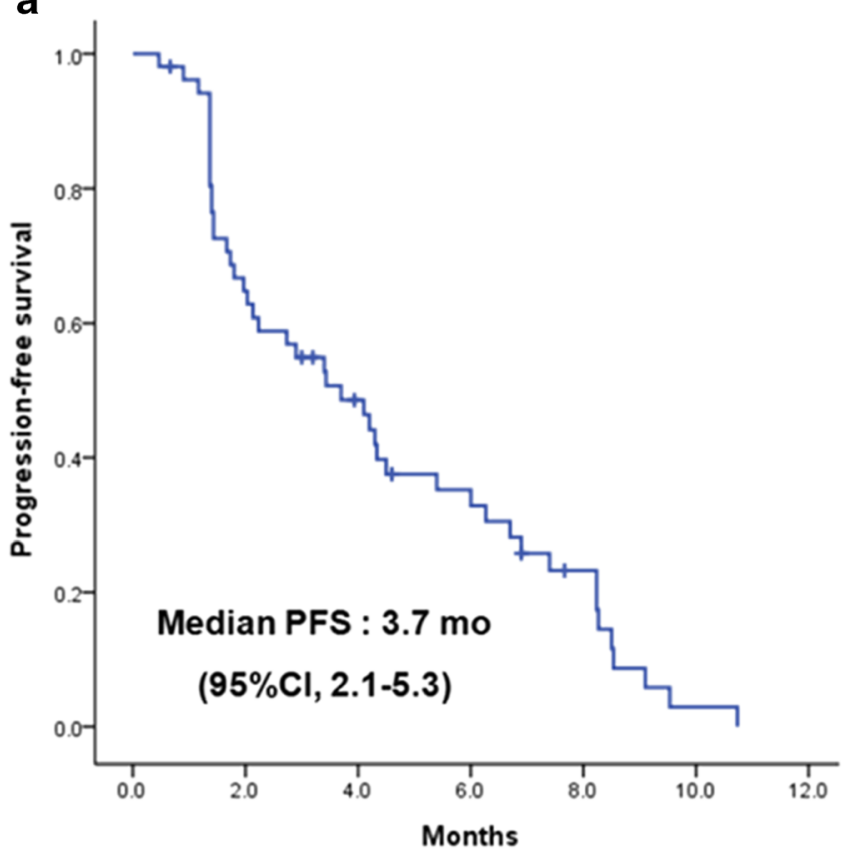

C

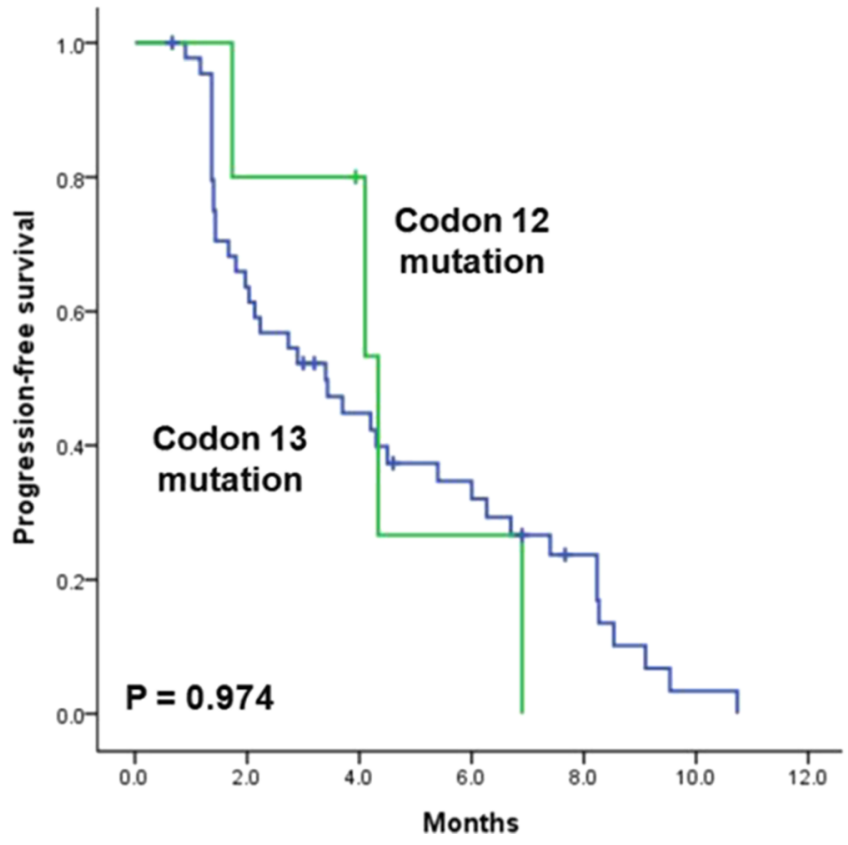

b

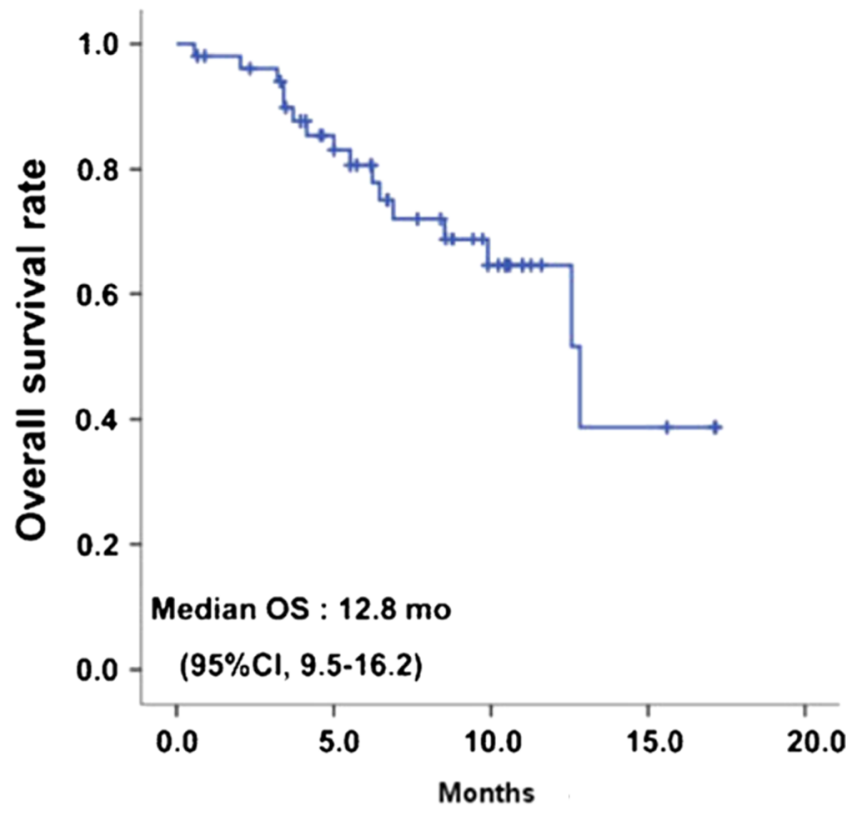

Fig. 2 a Progression-free survival b Overall survival c Overall survival according to codon 12 and codon 13 KRAS mutations 